\title{
Quantization of Klein-Gordon Scalar Field in Cosmological
}

\section{Inertial Frame}

\author{
Sangwha-Yi* \\ Department of Math, Taejon University 300-716, South Korea
}

*Corresponding Author: Sangwha-Yi, Department of Math, Taejon University 300-716, South Korea

Abstract: In the Cosmological Special Theory of Relativity, we quantized Klein-Gordon scalar field. We treat Lagrangian density and Hamiltonian in quantized Klein-Gordon scalar field in the Cosmological Special Theory of Relativity

Keywords: Cosmological inertial frame; Klein-Gordon scalar field; Hamiltonian; Quantization PACS Number: 03.30, 41.20

\section{INTRODUCTION}

Our article's aim is that we make quantization of Klein-Gordon scalar field in Cosmological Special Theory of Relativity (CSTR).

At first, space-time relations are in cosmological special theory of relativity (CSTR).[1]

$\left.c t=\gamma\left(c t+\frac{V_{0}}{c} \Omega^{2}\left(t_{0}\right) x^{\prime}\right), x \Omega\left(t_{0}\right)=\gamma \Omega\left(t_{0}\right) x+v_{0} \Omega\left(t_{0}\right) t^{\prime}\right)$

$\begin{aligned} & \Omega\left(t_{0}\right) y=\Omega\left(t_{0}\right) y^{\prime}, \\ & \Omega\left(t_{0}\right) z=\Omega\left(t_{0}\right) z^{\prime}\end{aligned}, \gamma=1 / \sqrt{1-\frac{V_{0}^{2}}{C^{2}} \Omega^{2}\left(t_{0}\right)}, \quad t_{0}$ is cosmological time

Proper time is

$$
\begin{aligned}
& d \tau^{2}=d t^{2}-\frac{1}{c^{2}} \Omega^{2}\left(t_{0}\right)\left[d x^{2}+d y^{2}+d z^{2}\right] \\
& =d t^{R}-\frac{1}{c^{2}} \Omega^{2}\left(t_{0}\right)\left[d x^{R}+d y^{R}+d z^{R}\right], t_{0} \text { is cosmological time }
\end{aligned}
$$

Angular frequency-wave number relation is in CSTR.

$$
\begin{aligned}
& \omega^{\prime}=\gamma\left(\omega-v_{0} \Omega\left(t_{0}\right) k_{1}\right), k_{1}^{\prime}=\gamma\left(k_{1}-\frac{V_{0}}{c^{2}} \Omega\left(t_{0}\right) \omega\right) \\
& k_{2}^{\prime}=k_{2}, k_{3}^{\prime}=k_{3}, \gamma=1 / \sqrt{1-\frac{V_{0}^{2}}{c^{2}} \Omega^{2}\left(t_{0}\right)}
\end{aligned}
$$

\section{Quantization of KLein-Gordon Scalar Field in CSTR}

Lagrangian density of Klein-Gordon scalar field in CSTR, 
$L=-\frac{1}{2}\left[-\left(\frac{1}{C} \frac{\partial \phi}{\partial t}\right)^{2} \Omega\left(t_{0}\right)+\frac{1}{\Omega\left(t_{0}\right)} \vec{\nabla} \phi \cdot \vec{\nabla} \phi+\frac{m_{0}{ }^{2} C^{2}}{\hbar^{2}} \phi^{2}\right]$

Hence, Euler-Lagrange equation is in CSTR,

$$
\left.\partial_{\mu}\left[\frac{\partial L}{\left.\partial \partial_{\mu} \phi\right)}\right]-\frac{\partial L}{\partial \phi}=\left[\Omega\left(t_{0}\right) \frac{1}{C^{2}}\left(\frac{\partial}{\partial t}\right)^{2}-\frac{1}{\Omega\left(t_{0}\right)} \nabla^{2}+\frac{m_{0}{ }^{2} C^{2}}{\hbar^{2}}\right] \phi=0\right)
$$

Hamiltonian of Klein-Gordon scalar field is in CSTR,

$$
H=\frac{1}{2}\left[\left(\frac{1}{C} \frac{\partial \phi}{\partial t}\right)^{2} \Omega\left(t_{0}\right)+\frac{1}{\Omega\left(t_{0}\right)} \vec{\nabla} \phi \cdot \vec{\nabla} \phi+\frac{m_{0}{ }^{2} C^{2}}{\hbar^{2}} \phi^{2}\right]
$$

The Klein-Gordon scalar field is divided by positive frequency mode and negative frequency mode.

$$
\phi(x)=\phi^{(+)}(x)+\phi^{(-)}(x)
$$

The positive frequency mode is

$$
\phi^{(+)}(x)=\int \frac{d^{3} k}{\left[(2 \pi)^{3} 2 \omega_{k}\right]^{\frac{1}{2}}} a(k) f_{k}(x)
$$

The negative frequency mode is

$$
\phi^{(-)}(x)=\int \frac{d^{3} k}{\left[(2 \pi)^{3} 2 \omega_{k}\right]^{\frac{1}{2}}} a^{(+)}(k) f_{k}(x)
$$

In this time, $f_{k}(x)$ is

$$
f_{k}(x)=\frac{1}{\left[(2 \pi)^{3} 2 \omega_{k}\right]^{\frac{1}{2}}} \exp \left[i\left(\frac{\omega_{k} t}{\sqrt{\Omega\left(t_{0}\right)}}-\vec{k} \cdot \vec{x} \sqrt{\Omega\left(t_{0}\right)}\right)\right]
$$

In this time,

$$
\frac{\omega_{k}}{C}=\left(k^{2}+\frac{m_{0}^{2} C^{2}}{\hbar^{2}}\right)^{\frac{1}{2}}
$$

Quantization of complex scalar field is in CSTR,

$$
\begin{aligned}
& \phi(x)=\int \frac{d^{3} k}{(2 \pi)^{3} 2 \omega_{k}}\left[a(k) \exp \left\{i\left(\frac{\omega_{k} t}{\sqrt{\Omega\left(t_{0}\right)}}-\vec{k} \cdot \vec{x} \sqrt{\Omega\left(t_{0}\right)}\right)\right\}\right] \\
& +\int \frac{d^{3} k}{(2 \pi)^{2} 2 \omega_{k}}\left[b^{+}(k) \exp \left\{-i\left(\frac{\omega_{k} t}{\sqrt{\Omega\left(t_{0}\right)}}-\vec{k} \cdot \vec{x} \sqrt{\Omega\left(t_{0}\right)}\right)\right\}\right] \\
& \phi^{+}(x)=\int \frac{d^{3} k}{(2 \pi)^{3} 2 \omega_{k}}\left[b(k) \exp \left\{i\left(\frac{\omega_{k} t}{\sqrt{\Omega\left(t_{0}\right)}}-\vec{k} \cdot \vec{x} \sqrt{\Omega\left(t_{0}\right)}\right)\right\}\right]
\end{aligned}
$$




$$
+\int \frac{d^{3} k}{(2 \pi)^{2} 2 \omega_{k}}\left[a^{+}(k) \exp \left\{-i\left(\frac{\omega_{k} t}{\sqrt{\Omega\left(t_{0}\right)}}-\vec{k} \cdot \vec{x} \sqrt{\Omega\left(t_{0}\right)}\right)\right\}\right]
$$

Hence, Hamiltonian $\mathrm{H}$ is in CSTR,

$$
H=\int \frac{d^{3} k}{(2 \pi)^{3} 2 \omega_{k}}\left[a^{+}(k) a(x)+b^{+}(k) b(k)\right]
$$

In this time,

$$
\begin{aligned}
& {\left[a(k), a^{+}\left(k^{\prime}\right)\right]=(2 \pi)^{3} 2 \omega_{k} \delta^{3}\left(\vec{k}-\vec{k}^{\prime}\right)} \\
& {\left[b(k), b^{+}\left(k^{\prime}\right)\right]=(2 \pi)^{3} 2 \omega_{k} \delta^{3}\left(\vec{k}-\vec{k}^{\prime}\right)}
\end{aligned}
$$

\section{CONCLUSION}

We quantized Klein-Gordon scalar field in CSTR. We treat Lagranian density and Hamiltonian.

\section{REFERENCES}

[1]S.Yi, "Cosmological Special Theory of Relativity", International Journal of Advanced research in Physical Science,7,11(2020),pp4-9

[2]S.Yi, "Klein-Gordon Equation and Wave Function in Cosmological Special Theory of Relativity", International Journal of Advanced research in Physical Science,7,12(2020),pp4-6

[3]C.Itzykson \& J.B.Zuber, Quantum Field Theory, McGraw-Hill, (1980)

[4]S.S.Schweber, An Introduction to Relativistic Quantum Field Theory, Haper and Raw, (1962)

[5]G.Kallen, Quatum Electrodynamics, Springer-Verlag, (1972)

[6]T.D.Lee, Particle Physics and Introduction to Field Theory, Ch. 2 Harwood Academic Publishers, (1981)

[7]L.H.Ryder, Quantum Field Theory, Cambridge University Press, (1985)

[8]J.D.Bjorken and S.D.Drell, Relativistic Quantum Fields, McGraw-Hill Co., New York, (1980)

Citation: Sangwha-Yi (2021). Quantization of Klein-Gordon Scalar Field in Cosmological

Frame. International Journal of Advanced Research in Physical Science (IJARPS) 8(2), pp.27-29, 2021.

Copyright: () 2021 Authors, This is an open-access article distributed under the terms of the Creative Commons Attribution License, which permits unrestricted use, distribution, and reproduction in any medium, provided the original author and source are credited. 